\title{
An explicit solution for calculating optimum spawning stock size from Ricker's stock recruitment model
}

Mark Scheuerell

Stock-recruitment models have been used for decades in fisheries management as a means of formalizing the relationship between parents and offspring. In particular, Ricker's stock recruitment model is widely used to describe the relationship between spawning fish and the expected number of surviving offspring. After model fitting, the spawning stock size that produces the maximum sustainable yield $\left(S_{M S Y}\right)$ to a fishery, and the harvest corresponding to it $\left(U_{M S Y}\right)$, are two of the most common biological reference points of interest to fisheries managers. However, to date there has been no explicit solution for either reference point because of the transcendental nature of the equation needed to solve for them. Therefore, numerical or statistical approximations have been used for more than 30 years. Here I provide explicit formulae for calculating both $S_{M S Y}$ and $U_{M S Y}$ in terms of the productivity and density-dependent parameters from Ricker's model. 
1 An explicit solution for calculating optimum spawning stock size from 2 Ricker's stock recruitment model

3 Mark D. Scheuerell

4 Fish Ecology Division, Northwest Fisheries Science Center, National Marine Fisheries Service, National

5 Oceanic and Atmospheric Administration, Seattle, WA 98112 USA, mark.scheuerell@noaa.gov

7 


\section{ABSTRACT}

9 Stock-recruitment models have been used for decades in fisheries management as a

10 means of formalizing the expected number of offspring that recruit to a fishery based on the

11 number of parents. In particular, Ricker's stock recruitment model is widely used due to its

12 flexibility and ease with which the parameters can be estimated. After model fitting, the

13 spawning stock size that produces the maximum sustainable yield $\left(S_{M S Y}\right)$ to a fishery, and the

14 harvest corresponding to it $\left(U_{M S Y}\right)$, are two of the most common biological reference points of

15 interest to fisheries managers. However, to date there has been no explicit solution for either

16 reference point because of the transcendental nature of the equation needed to solve for them.

17 Therefore, numerical or statistical approximations have been used for more than 30 years. Here I

18 provide explicit formulae for calculating both $S_{M S Y}$ and $U_{M S Y}$ in terms of the productivity and

19 density-dependent parameters of Ricker's model. 


\section{INTRODUCTION}

21 One of the most difficult problems in the assessment of fish stocks is establishing the

22 relationship between the spawning stock and subsequent recruitment (Hilborn and Walters

23 1992). Stock-recruitment models have been used for decades in fisheries management as a means

24 of formalizing this relationship (Beverton and Holt 1957; Ricker 1954). Over time, a variety of

25 functional forms have emerged to capture varying assumptions about depensatory and

26 compensatory mortality (Hilborn and Walters 1992). In a classroom setting, deterministic

27 versions of the models provide useful constructs for teaching about management reference points

28 such as maximum sustained yield (MSY).

29 In particular, Ricker's stock recruitment model (Ricker 1954; Ricker 1975) is one of the

30 most widely used models to describe the population dynamics of fishes, such that

$$
R=\alpha S e^{-b S}
$$

$32 R$ is the number of recruits produced, $S$ is the number of spawners, $\alpha$ is the dimensionless

33 number of recruits per spawner produced at very low spawner density, and $b$ is the strength of

34 density dependence (units: spawner-1). It is common to substitute $\alpha=e^{a}$ into equation (1) and

35 rewrite it as

$$
R=S e^{a-b S}
$$

37 To make the model reflect a stochastic process, equation (2) is typically multiplied by a log-

38 normal error term, so that

$$
R=S e^{a-b S} e^{\varepsilon},
$$

and $\varepsilon$ is a normally distributed error term with a mean of $-1 / 2 \sigma$ and variance $\sigma \square \square \square$ his non-zero

41 mean ensures that $a$ is interpreted as the mean recruits per spawner rather than the median 
42 (Hilborn 1985). Part of the model's popularity is due to the relative ease with which its 43 parameters are estimated. After log transformation, equation (3) is typically rewritten as

$$
\ln (R / S)=a-b S+\varepsilon
$$

and the parameters are estimated via simple linear regression. I note here that estimation of the parameters via a simple observation-error model like (4) can lead to substantial biases in $a$ and $b$

47 if the sample size is low $(n \leq 10)$ due to autocorrelation in the residuals $\varepsilon$ (Walters 1985).

parameters can be used to derive various biological reference points of interest to fisheries managers. Some of these metrics are rather trivial to compute. For example, the spawning stock size leading to maximum recruit production $\left(S_{M S R}\right)$ is simply $1 / b$. However, other reference points are much less straightforward to calculate. In particular, the spawning stock expected to produce the maximum sustainable yield $\left(S_{M S Y}\right)$ under deterministic dynamics is of common

54 interest.

$$
Y=R-S=S e^{a-b S}-S,
$$

57 and then take the derivative of $Y$ with respect to $S$ :

$$
\frac{d Y}{d S}=(1-b S) e^{a-b S}-1
$$

$S_{M S Y}$ is then determined by setting equation (6) to zero and solving for $S$. Upon initial inspection,

60 however, there does not appear to be an explicit solution to this equation in terms of $S$, and

61 therefore $S_{M S Y}$ is typically solved "by trial" (Ricker 1975) with some form of gradient method 62 (e.g., Newton's as in Hilborn 1985). 

model whereby the ratio of spawning stock size at MSY to that at the unfished equilibrium $65\left(S_{M S Y} / S_{r}\right)$ is a linear function of the parameter $a$. Specifically, for $0<a \leq 3$ he estimated that

$$
\begin{aligned}
& \frac{S_{M S Y}}{S_{r}}=\frac{S_{M S Y}}{(a / b)}=0.5-0.07 a, \text { and } \\
& S_{M S Y}=\frac{a(0.5-0.07 a)}{b} .
\end{aligned}
$$

Although this approximation is very useful due to its simplicity, there is no underlying fundamental support for the statistical form of the relationship.

\section{METHODS}

Here I make use of the Lambert W function, $W(z)$, to demonstrate an explicit solution to

equation (4) that precludes the need to estimate $S_{M S Y}$ via numerical methods or Hilborn's (1985) that satisfies

$$
W(z) e^{W(z)}=z
$$

for any complex number $z$ (Lambert 1758 and Euler 1783 as cited in Corless et al. 1996). Here we are interested only in real values, however, so I replace $z$ with $x$ and note that $W(x)$ is only defined for $x \geq-1 / e$ (Corless et al. 1996). Furthermore, this function is not injective and has two values for $-1 / e \leq x \leq 0$, but as I show below, we are concerned only with the region where $x>0$ and $W(x)$ is a singular, non-negative value. I begin my explicit solution of $S_{M S Y}$ by setting equation (6) to zero, such that

$$
\left(1-b S_{M S Y}\right) e^{a-b S_{M S Y}}=1
$$


85 After rearranging terms and multiplying both sides by $e$, we arrive at

86

$$
\left(1-b S_{M S Y}\right) e^{1-b S_{M S Y}}=e^{1-a}
$$

87 At this point I note the relationship between equations (10) and (8), with $1-b S_{M S Y}=W(x)$ and $88 e^{1-\mathrm{a}}=z$. Therefore, we can write

$$
1-b S_{M S Y}=W\left(e^{1-a}\right), \text { and hence }
$$

$$
S_{M S Y}=\frac{1-W\left(e^{1-a}\right)}{b} .
$$

91 We now have an explicit solution for $S_{M S Y}$ that depends only on the parameters $a$ and $b$ from equation (2). As mentioned above, $W(x)$ is only defined for $x \geq-1 / e$, which does not pose any problems here because $x=e^{1-a}>0 \forall a \in \mathbb{R}$. For visualization purposes, I show a plot of $W\left(e^{1-a}\right)$ versus $a$ in Fig. 1.

We can also derive an explicit formula for calculating the fraction of the return harvested at $S_{M S Y}$, which I call $U_{M S Y}$. As Ricker (1975) shows,

$$
U_{M S Y}=b S_{M S Y},
$$

and therefore substituting (12) into (13) gives

$$
U_{M S Y}=1-W\left(e^{1-a}\right)
$$

101 Corless et al. (1996) recommend Halley's method, with the update equation given by

$$
w_{j+1}=w_{j}-\frac{w_{j} e^{w_{j}}-x}{e^{w_{j}}\left(w_{j}+1\right)-\frac{\left(w_{j}+2\right)\left(w_{j} e^{w_{j}}-x\right)}{2 w_{j}+2}} .
$$

103 I used an initial guess of $w_{0}=3 / 4 \ln (x+1)$ based on the shape of $W(x)$ over the range of $a$ typically 104 considered in fisheries research (i.e., $0<\mathrm{a}<3$ as in Hilborn 1985). If, however, one must 
105 estimate $W(x)$ numerically, then one should ask whether doing so is, in fact, computationally

106 faster. Therefore, as a test I randomly selected 1000 values each for $0<a \leq 3$ and $10^{-5} \leq b \leq 10^{-3}$,

107 and then solved for $S_{M S Y}$ using both Newton's method as suggested by Ricker (1975), and

108 Halley's method as in equation (15).

109 RESULTS AND DISCUSSION

110 Recent analyses have relied on estimating $S_{M S Y}$ via Hilborn's (1985) linear approximation

111 when calculating optimal yield profiles (Fleischman et al. 2013) or the effects of observation

112 error on biases in parameter estimates ( $\mathrm{Su}$ and Peterman 2012). On the other hand, solving for

$113 S_{M S Y}$ using $W(x)$ and Halley's method is not only convenient; it also offers an appreciable

114 computational advantage over the standard Newton method. Although both methods converged

115 in less than 10 iterations during my test, Halley's method was always faster and less variable

116 overall (Fig. 2). Therefore, estimating $S_{M S Y}$ via Halley's method might save significant time in

117 applications such as management strategy evaluations that are much more computationally

118 intensive than a simple one-case solution.

119 Although implementing equation (15) may seem a bit daunting to individuals less

120 familiar with numerical methods, a variety of contemporary software packages (e.g., MATLAB,

$121 \mathrm{R})$ include built-in functions to calculate $W(x)$ directly. This means that anyone using a personal

122 computer to estimate the parameters in a Ricker model can easily estimate $S_{M S Y}$ from equation

123 (12) as I demonstrate in Table 1; I show the results from my R implementation for a range of $a$

124 and $b$ in Fig. 3. For those preferring to use Microsoft Excel, there is no built-in function to

125 calculate $W(x)$, but I have implemented equation (15) as the VBA function 'LAMBERTW' in the

126 Microsoft Excel add-in file 'LambertWfunc.xlam' (see Fig. S1 for download and install

127 instructions). 
Here I have outlined a new method to easily calculate $S_{M S Y}$ from the productivity $(a)$ and

129 density-dependent $(b)$ parameters in a Ricker model using readily available functions in several

130 software packages. This method is much more straightforward than trying to solve for $S_{M S Y}$ using

131 numerical methods and should be useful in many classroom settings. Although there could be

132 some utility in actually going through the exercise of numerically deriving the answer, it is rare

133 nowadays, for example, for anyone to code a random number generator because of their

134 ubiquitous implementation in standard software. In addition, the explicit analytical solution is

135 closed-form with respect to the special functions, and therefore precludes the need to estimate

$136 S_{M S Y}$ via Hilborn's (1985) approximation. Thus, due to the speed and ease with which these new

137 equations are calculated, I recommend that practitioners use them for the estimation of $S_{M S Y}$ and

$138 U_{M S Y}$ in lieu of those listed in Appendix III of Ricker (1975) and Table 7.2 of Hilborn and

139 Walters (1992).

\section{ACKNOWLEDGEMENTS}

141 I thank Jim Thorson, Jason Link, Brian Kennedy, Olaf Jensen, Ray Hilborn, Tim

142 Essington, Curry Cunningham, and Trevor Branch for helpful discussions and comments on the

143 manuscript.

\section{REFERENCES}

145 Beverton, R. J. H., and S. J. Holt. 1957. On the Dynamics of Exploited Fish Populations. Fishery

146 Investigations Series II Volume XIX, Ministry of Agriculture, Fisheries and Food.

147 Corless, R. M., G. H. Gonnet, D. E. G. Hare, D. J. Jeffrey, and D. E. Knuth. 1996. On the

148 Lambert W function. Advances in Computational Mathematics 5(4):329-359. 
149 Fleischman, S. J., M. J. Catalano, R. A. Clark, and D. R. Bernard. 2013. An age-structured state150 space stock-recruit model for Pacific salmon (Oncorhynchus spp.). Canadian Journal of $151 \quad$ Fisheries and Aquatic Sciences 70(3):401-414.

152 Hilborn, R. 1985. Simplified calculation of optimum spawning stock size from ricker stock recruitment curve. Canadian Journal of Fisheries and Aquatic Sciences 42(11):18331834.

155 Hilborn, R., and C. J. Walters. 1992. Quantitative Fisheries Stock Assessment: Choice, 156 Dynamics and Uncertainty. Chapman and Hall, New York.

157 McCoy, M. W., and B. M. Bolker. 2008. Trait-mediated interactions: influence of prey size, 158 density and experience. Journal of Animal Ecology 77(3):478-486.

159 Reluga, T. 2004. A two-phase epidemic driven by diffusion. Journal of Theoretical Biology 160 229(2):249-261.

161 Ricker, W. E. 1954. Stock and recruitment. Journal of the Fisheries Research Board of Canada 162 11(4):559-623.

Ricker, W. E. 1975. Computation and interpretation of biological statistics of fish populations.

164 Bulletin of the Fisheries Research Board of Canada 191:832 p.

165 Su, Z., and R. M. Peterman. 2012. Performance of a Bayesian state-space model of semelparous 166 species for stock-recruitment data subject to measurement error. Ecological Modelling 167 224(1):76-89.

168 Walters, C. J. 1985. Bias in the estimation of functional relationships from time series data.

169 Canadian Journal of Fisheries and Aquatic Sciences 42(1):147-149.

170 Wang, F. 2010. Application of the Lambert W function to the SIR epidemic model. The College Mathematics Journal 41(2):156-159. 
172 Table 1. Example code for directly calculating $S_{M S Y}$ in R, Matlab, and Excel; the values for $a$ and

$173 b$ were chosen arbitrarily. Note that the R code requires the 'gsl' package to be installed, and the

174 Excel code requires the 'LAMBERTW' function contained in the Excel Add-in file

175 LambertWfunc.xlam.

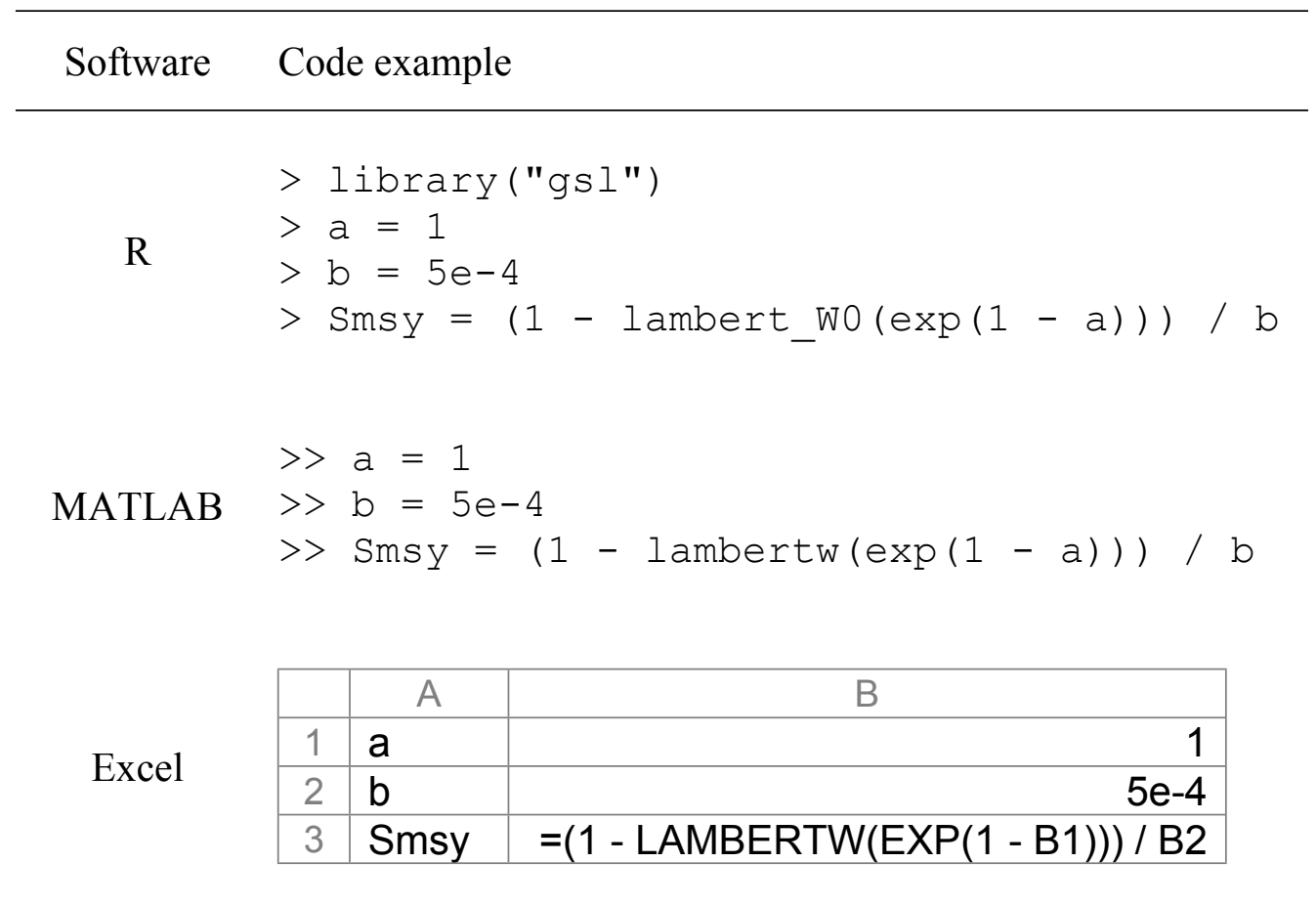

176 


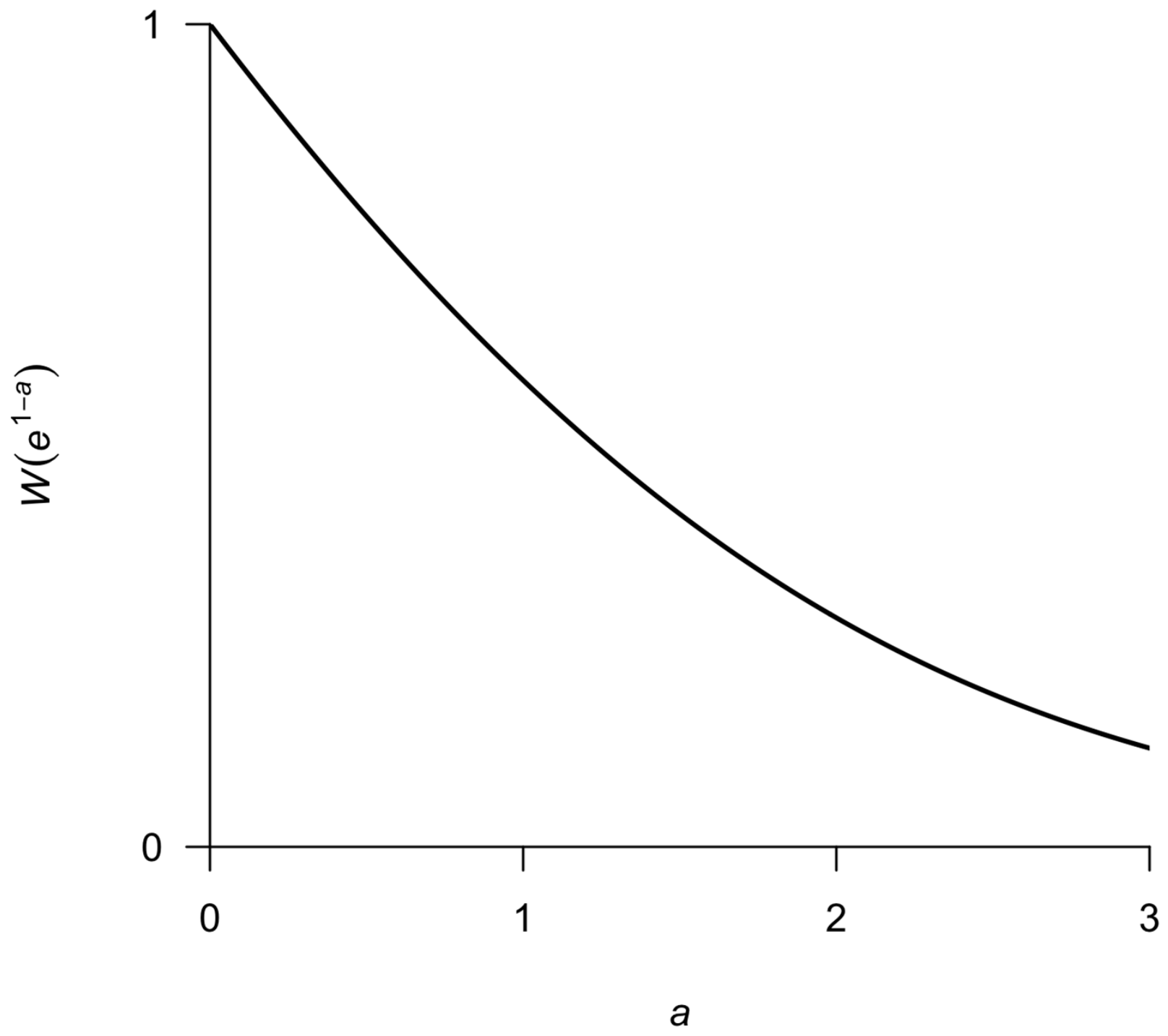

177

178 Figure 1. Plot of $W\left(e^{1-a}\right)$ over a range in values of $a$ typically encountered in fisheries. 


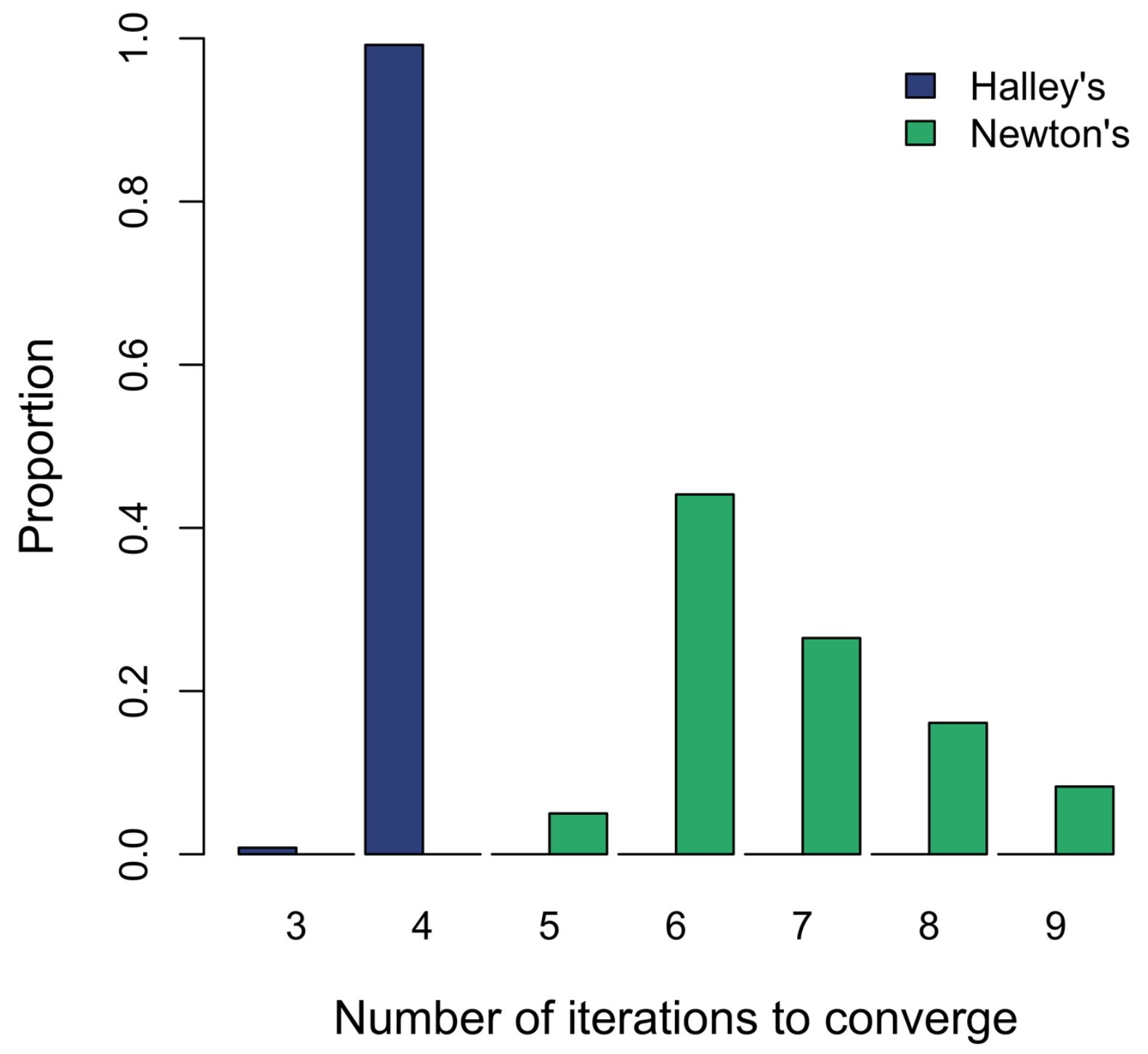

180 Figure 2. Histograms showing the distribution of the number of iterations that each of the two 181 numerical methods takes to converge to $S_{M S Y}$ using a threshold of $10^{-6}$. 


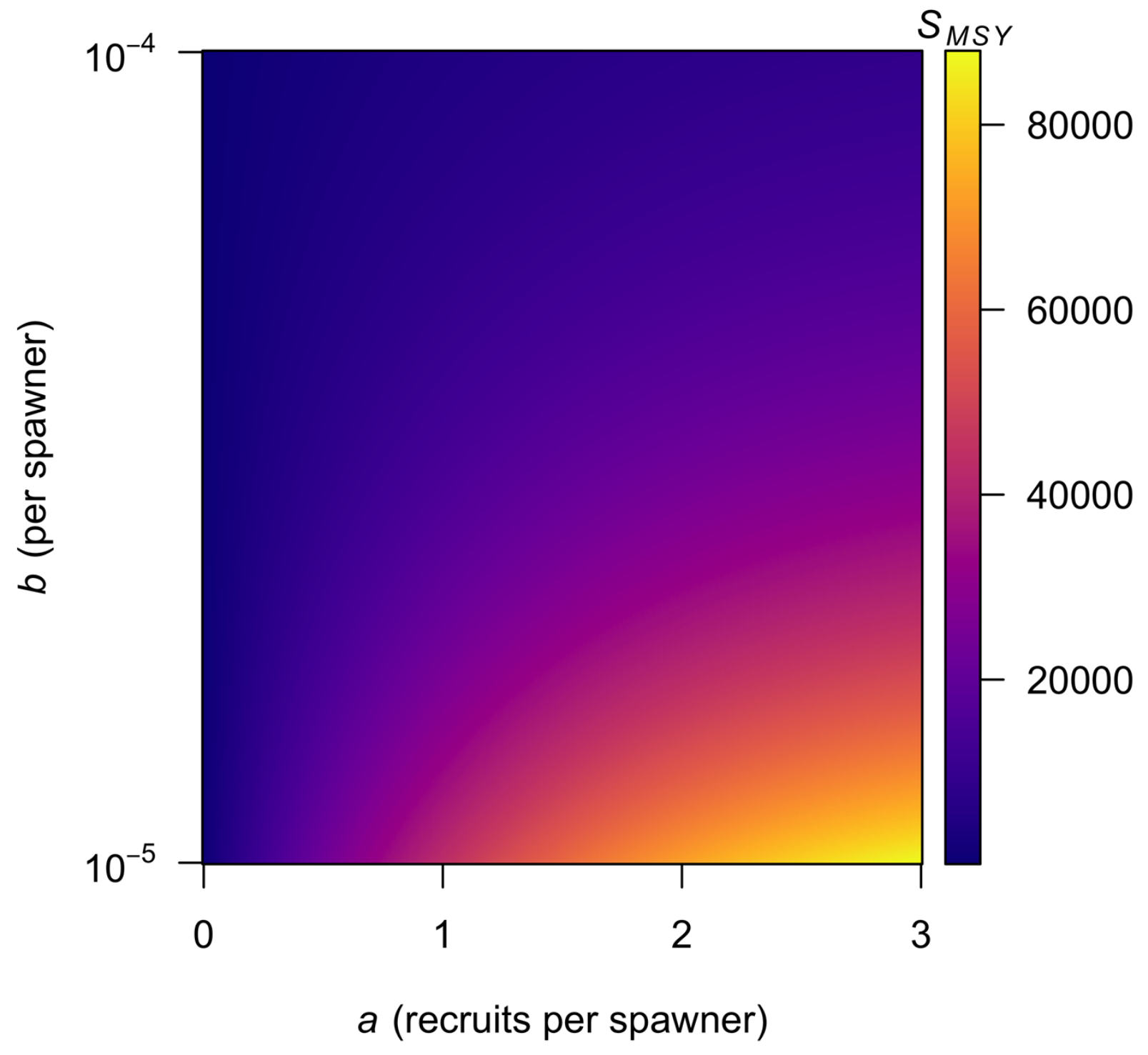

183 Figure 3. Contour plot showing values of $S_{M S Y}$ for combinations of the $a$ and $b$ parameters in 184 Equation (2). 